\title{
Sylvian Fissure Epidermoid Tumor Presenting With Intractable Non-Pulsatile Tinnitus
}

\author{
Rothaina Saeedia, Khalid Alqadi ${ }^{\mathrm{b}}$, Saleh Baeesa ${ }^{\mathrm{a}, \mathrm{c}}$
}

\begin{abstract}
Intracranial epidermoid cysts are benign congenital lesions of ectodermal origin. They account for approximately $1 \%$ of all intracranial tumors and commonly located in the cerebellopontine angle, parasellar region and subarachnoid spaces of the basal cisterns. We report a case of a 27-year-old female with a large epidermoid cyst located in the right Sylvian fissure who presented with a headache, hearing impairment and tinnitus. She underwent craniotomy and complete resection of the tumor with the resolution of the tinnitus. Such location and presentation are rarely encountered for epidermoid tumors.
\end{abstract}

Keywords: Epidermoid tumor; Tinnitus; Sylvian fissure

\section{Introduction}

Epidermoid tumors are congenital lesions formed during embryogenesis that comprise $1 \%$ of all intracranial tumors and they tend to grow slowly and remain asymptomatic till the third or fourth decade of age with no gender difference [1, 2]. These tumors are lined by stratified squamous epithelium and contain keratin, cholesterol, and cellular debris [3]. They are commonly encountered intracranially in the cerebellopontine and parasellar regions [3, 4]. The clinical manifestations are location-dependent and range from a headache and gait instability to obstructive hydrocephalus causing intracranial hypertension. Tinnitus is an unusual presentation of epidermoid tumors in the cerebellopontine angle (CPA) region but has never been described for Sylvian fissure location $[5,6]$.

We present a case of a 27-year-old female with a large epidermoid tumor located in the right Sylvian fissure who pre-

\footnotetext{
Manuscript accepted for publication February 18, 2017

aDivision of Neurosurgery, Faculty of Medicine, King Abdulaziz University, Jeddah, Saudi Arabia

bepartment of Neurosciences, King Faisal Specialist Hospital and Research Center, Jeddah, Saudi Arabia

${ }^{\mathrm{c} C}$ Corresponding Author: Saleh S. Baeesa, Division of Neurosurgery, Faculty of Medicine, King Abdulaziz University, PO Box 80215, Jeddah 21589, Saudi Arabia. Email: sbaeesa@kau.edu.sa
}

doi: https://doi.org/10.14740/jnr422e sented with an occipital headache, right-sided tinnitus, and decrease of hearing in the right ear.

\section{Case Report}

A 27-year-old female patient with no past medical history of significance presented with a 2-year history of a mild paroxysmal headache. She was evaluated in a polyclinic and diagnosed as a migraine headache and started with anti-migraine therapy with subjective improvement. The type of her headache has changed over few months and became persistent, located in the occipital regions, and associated with a bilateral earache that was worse on the right and triggered by noise with subjective decreased hearing in the right ear. Her symptoms included non-positional, non-pulsatile tinnitus which became constant and disturbed her sleep and daily life activities. She was evaluated by an otologist who confirmed normal local ear examination and her audiogram was normal.

On presentation to our center, her vital signs and general physical exam were normal. The neurological exam did not reveal any focal neurological deficits, and there was no papilledema. Magnetic resonance imaging (MRI) scan revealed a large expanding and lobulated cystic lesion in the right Sylvian fissure insinuating into the frontal, insular, and temporal lobes (Fig. 1). On magnetic resonance angiography (MRA), the cyst was encasing, without compromising, the branches of the right middle cerebral artery (MCA). The cyst was hypointense and hyperintense in T1 and T2-wiegthed images, respectively, and hyperintense in the fluid-attenuated inversion recovery (FLAIR) and the diffusion-weighted (DW) sequences. The lesion has demonstrated no enhancement following intravenous contrast administration; these findings are consistent with the diagnosis of the epidermoid tumor.

The patient started on intravenous dexamethasone. Then, she underwent a right frontotemporal craniotomy. At surgery, a thick whitish membrane was encountered that contains pearly white contents, classically encountered in epidermoid tumors (Fig. 2). The MCA branches were found encased, and adherent to the cyst wall and great effort was achieved for adequate separation and removal of the cyst wall. After the microscopic evacuation of the cystic contents, the cyst's wall was dissected from the surrounding brain tissue, and blood vessels and complete excision were achieved.

The histopathological exam revealed the characteristic 

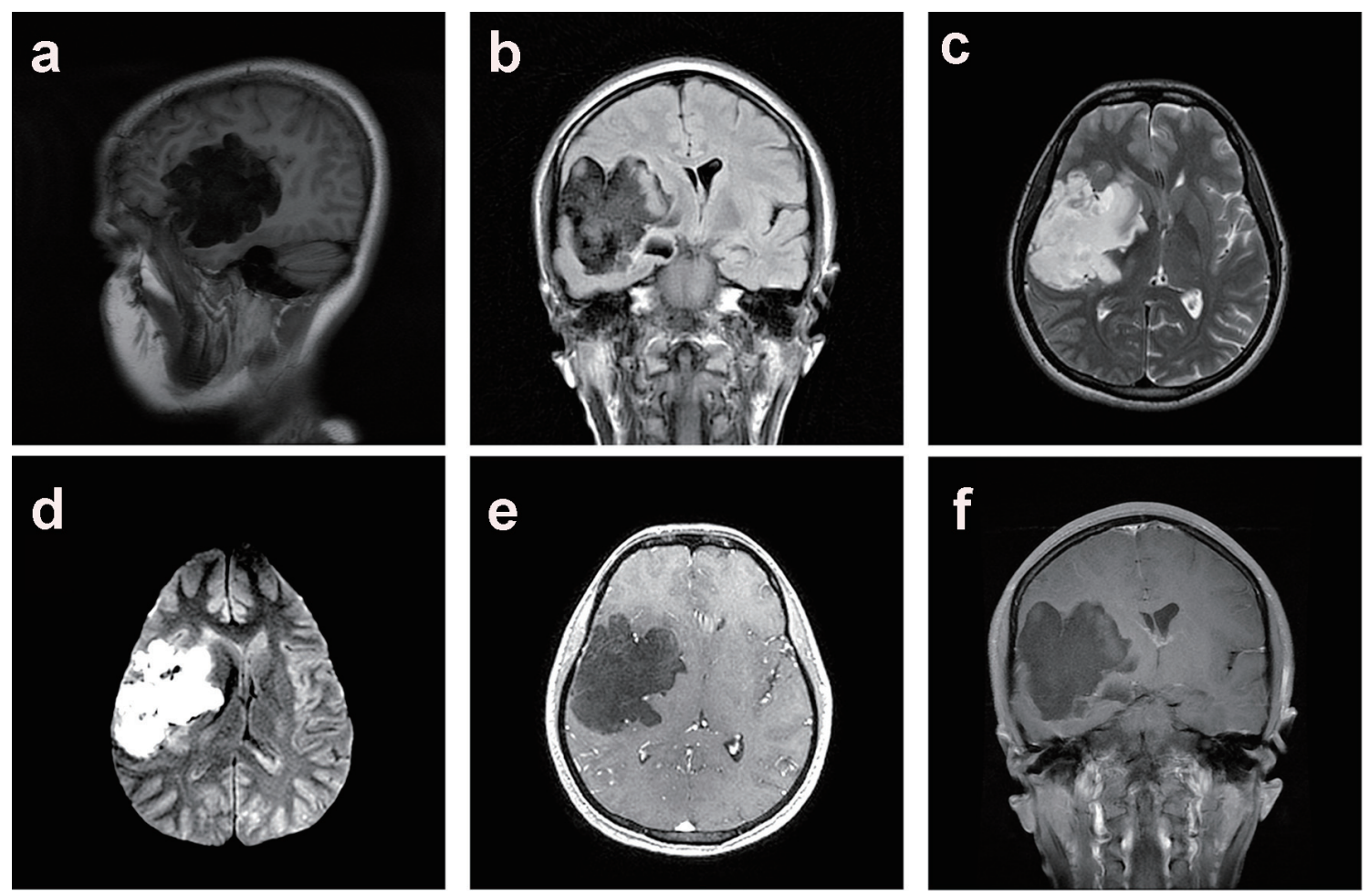

Figure 1. Preoperative MRI scan of the brain demonstrated a large right Sylvian epidermoid tumor. The lesion demonstrated a hypointense signal on para-sagittal T1-WI (a) and coronal (b) T2-FLAIR sequences, hyperintense signal on axial T2-WI (c) and axial DWI (d) MRI scan. There was no enhancement of the hypointense lesion following IV contrast as demonstrated on axial (e) and coronal (f) T1-WI MRI scan.

features of the epidermoid tumor (Fig. 3).

The patient had a smooth postoperative recovery with complete resolution of previous symptoms, particularly the tinnitus. She had at 24-h and 1-year a follow-up MRI scan which confirmed complete resection with no recurrence or vascular insults (Fig. 4).

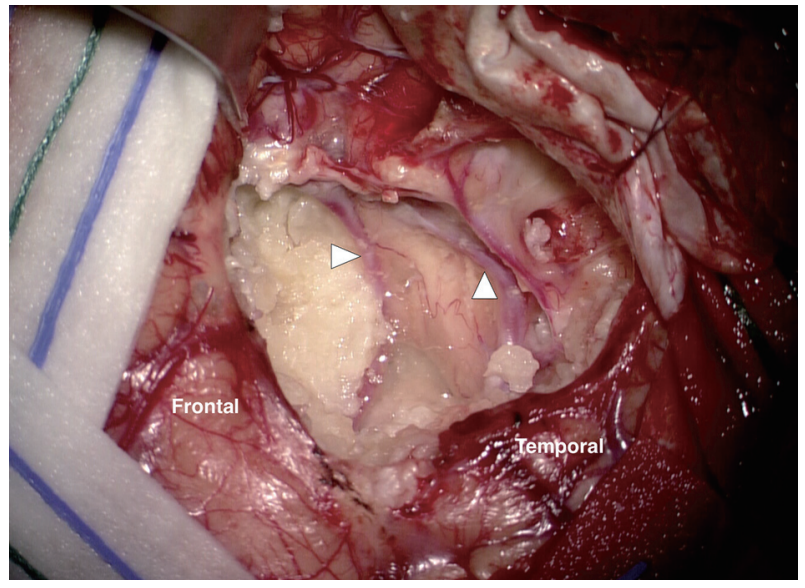

Figure 2. Microscopic photograph at surgery after right frontotemporal craniotomy and dural opening demonstrating an epidermoid tumor with characteristic "pearl" contents. The middle cerebral artery branches were found displaced and adherent to the wall of the cystic tumor (arrowheads).

\section{Discussion}

Epidermoid cysts are a benign tumor with slow growth that tends to envelop neurovascular structure without displacement [1]. Compression on these structures triggers the symp-

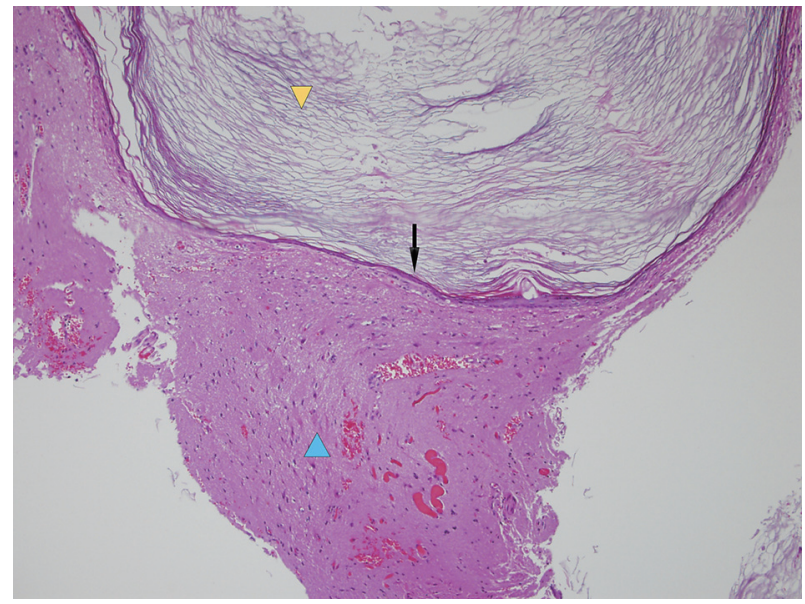

Figure 3. Histopathological microphotograph demonstrating a stratified epithelium wall (black arrow) containing keratin and cholesterol (yellow arrowhead) characteristics of an epidermoid cyst, and surrounding inflammatory cells and gliosis (blue arrowhead) (hematoxylin and eosin stain, $\times 100$ magnification). 

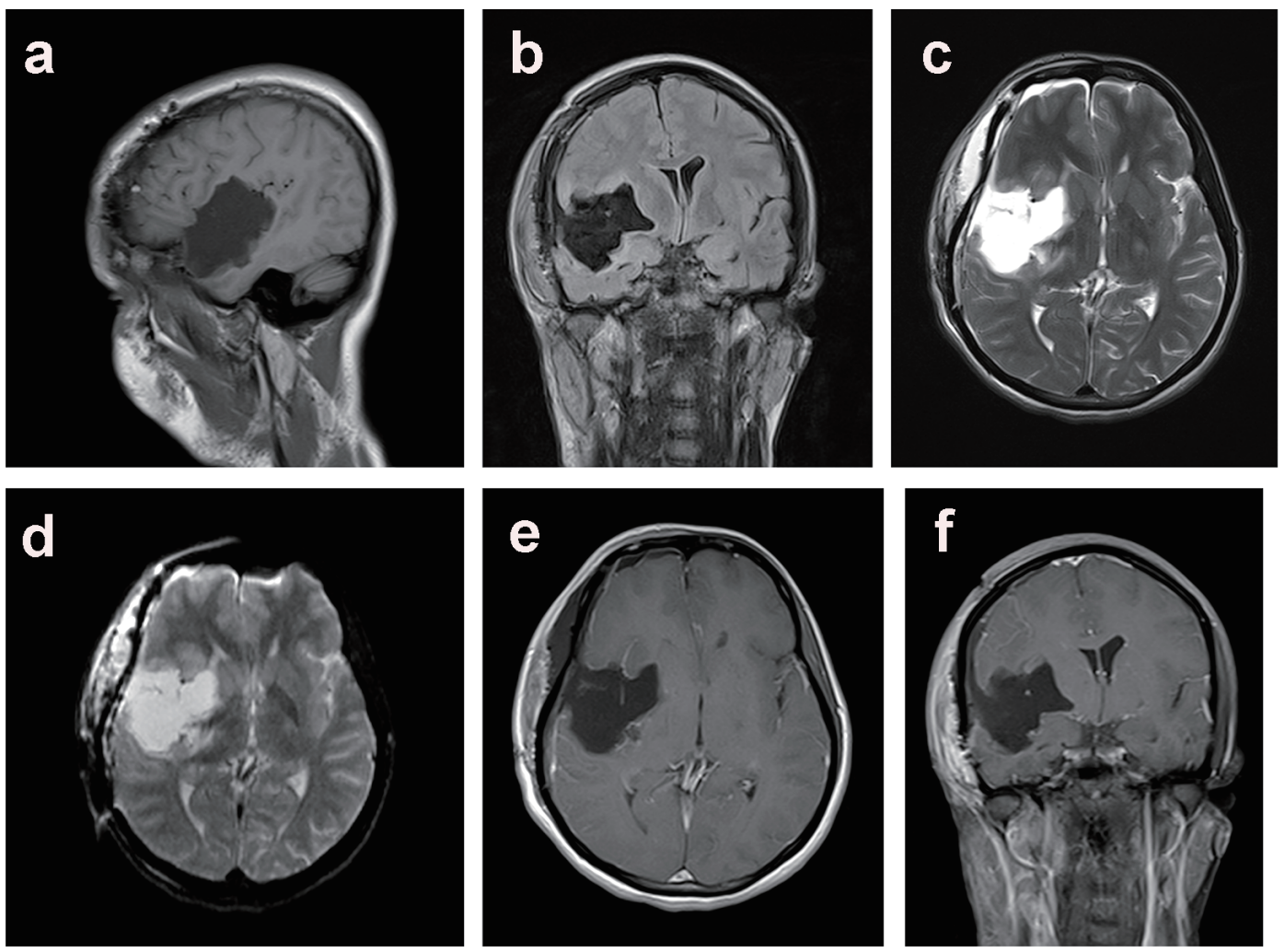

Figure 4. Postoperative MRI scan of the brain at 1 year follow up demonstrated complete resection of the epidermoid tumor as evidenced as a hypointense signal on para-sagittal T1-WI (a) and coronal (b) T2-FLAIR sequences, marked decrease hyperintense signal on axial T2-WI (c) and axial DWI (d) images. There were no vascular complications or enhancement of the hypointense lesion following IV contrast as demonstrated on axial (e) and coronal (f) T1-WI MRI scan.

toms; often they cause irritation of the cranial nerve resulting in cranial nerve hyperactive dysfunction, such as trigeminal or glossopharyngeal neuralgia, or hemifacial spasm and also may present symptoms of cranial nerve, cerebellar and brain stem dysfunction, as well as hydrocephalus and meningeal irritation [4]. There are many theories regarding the origin of epidermoid tumors; these have included embryonic inclusions, trauma, and differentiation from multipotential cell rests and epithelial remnants [3]. They are most often found in the CPA area, while rarely seen in the Sylvian fissure [2]. In this case, the patient had Sylvian fissure epidermoid tumor, manifested with off and on occipital headache, right more than left ear pain that triggered by noise, as well as vomiting and a subjective decrease of hearing in the right ear. These symptoms were manageable, but what bothered the patient the most was the non-positional, non-pulsatile constant tinnitus.

Epidermoid cyst diagnosis is made most of the time based on characteristic image findings on by CT scan or MRI [7]. In this patient, the lesion was a large lobulated cystic lesion filling and expanding the right Sylvian fissure and insinuating through the right frontal and temporal lobes; it was exerting mass effect on the right basal ganglia, thalamus and brain stem with midline shift by $8 \mathrm{~mm}$, and encasing the branches of the right MCA without obstruction. The most relevant differential diagnosis for epidermoid cyst is arachnoid cysts, neuroenteric cyst, and cystic neoplasms. They can usually be differentiated by MRI of the brain. Conventional MRI sometime cannot reliably be used to distinguish epidermoid tumors from arachnoid, and neuroenteric cysts since all lesions are hypointense about brain parenchyma on T1-weighted MRI and hyperintense on T2-weighted images. In contrary, FLAIR and DW sequences can efficaciously be used for diagnosis of epidermoid cysts by revealing its solid nature. DW imaging is superior to other MRI sequences in delineating the borders of the epidermoid cyst. FLAIR MRI is based on the nulling of the signal from CSF. Epidermoid tumors characteristically present on this sequence as heterogeneous lesions with central parts of the tumor being hyperintense about the hypointense CSF. The presence of enhancement after intravenous administration of contrast is usually in favor of cystic neoplasms.

Histopathology of the tumor revealed the characteristic features of an epidermoid tumor and the differential diagnosis includes dermoid cyst and arachnoid cyst. Despite the good availability of conventional MRI, the diagnosis of epidermoid cyst can be a challenge, probably due to low incidence [7].

The pathophysiology of tinnitus is still incompletely understood but there is increasing evidence that tinnitus is due to alterations in neuronal functioning in the central auditory system. Tinnitus as a phantom auditory perception seems to be the correlate of maladaptive attempts of the brain at reorganization, due to distorted sensory input [8]. It is described as an 
unpleasant sound sensation without external stimulation.

The most recent pathophysiologic theory suggests that the central nervous system is the generator of tinnitus. And, according to some literatures within the neurological field, a loss of cochlear input to neurons in the central auditory system (such as occurs in cochlear hair cell damage or a lesion of the vestibulocochlear nerve) can result in abnormal neural activity in the auditory cortex [9]. This activity is related to the perception of tinnitus. There is also a loss of suppression of the neural feedback loops which help tune and reinforce auditory memory in the central auditory cortex. Moreover, a disruption of this feedback loop leads to the disinhibition of normal synapses and creation of uncontrolled alternative neural synapses which lead to the abnormal auditory perception of tinnitus $[9,10]$.

In neurophysiology, the complex pathophysiologic mechanisms involved tinnitus, elucidating sites in which these processes take place. It is now known that approximately $24 \%$ of cases occur as a result of abnormalities within the otoacoustic periphery (i.e. inner ear and the vestibulocochlear nerve), 35\% originate from the acoustic pathways, and $41 \%$ have their cause within supratentorial structures [11]. A decrease in inhibition and increase in excitation may lead to an excitatory-inhibitory imbalance causing neuronal hyperexcitability in these regions and lead to the perception of tinnitus. However, neuronal excitability can be modulated by different neurotransmitters and neuromodulators that act on voltage- or ligand-gated channels, thus providing potential pharmacologic targets [10].

Our patient's main complaint was tinnitus, which showed aggravation during her completion of the audiological and electrophysiological evaluation. We believe that a pressure effect following a spontaneous cyst enlargement might also be the reason of tinnitus, as tinnitus is an active physical process occurring in multiple neural substrates in response to the peripheral or central stimulus.

A literature review shows that it is often difficult to interpret the relationship between inner ear symptoms and intracranial arachnoid cysts. In fact, there is a debate whether arachnoid cysts are associated with these symptoms in most cases $[2,3]$.

Arachnoid cysts most frequently occur in the middle cranial fossa. The last histopathologic study, the rare cases of arachnoid cysts confined in the internal auditory canal, has been reported [10]. A common hypothesis for primary arachnoid cysts genesis is arachnoid tearing after complete differentiation in the 15 th week of gestation or during separation between arachnoid membrane and dura mater. In spite of the fact that these lesions present symptoms during childhood, they may give symptoms later in adulthood, or remain asymptomatic [12].

Symptoms might be caused by a space-occupying effect with pressure on surrounding structures, a change in CSF dynamics, or dysgenesis of the brain. Late symptoms presentation is attributed to intracystic bleeding and subdural hematoma following head trauma, or to spontaneous cyst expansion. A wide range of symptoms at the initial patient's evaluation have been reported which were seemingly unrelated to the location of the cyst $[12,13]$.

Thus, patients may present with atypical signs and symp- toms such as sleep apnea, nausea, vomiting, tinnitus, unsteadiness, and vertigo, which initially may not attribute to central nervous system lesions and therefore adequate management is delayed [12]. We suggest that inner ear symptoms might be attributed to middle fossa arachnoid cysts. In such cases, complete clinical and electrophysiological examinations are needed, and any pathological finding should be carefully evaluated for a prompt diagnosis [10].

\section{Conclusion}

Epidermoid cysts may present with non-specific complaints such as triggered ear pain and tinnitus. Symptoms related to large epidermoid cysts, in most cases, respond well to excision of the cyst. We encouraged stressing the importance of early diagnosis and intervention in these rare tumors.

\section{Disclosure}

The authors have no conflicts of interest. All authors have approved the final article.

\section{References}

1. Shah A, Makkiyah F, Goel A. Sylvian fissure epidermoid cyst presenting with intention tremor. Asian J Neurosurg. 2016;11(2):174-175.

2. Son DW, Choi CH, Cha SH. Epidermoid tumors in the cerebellopontine angle presenting with trigeminal neuralgia. J Korean Neurosurg Soc. 2010;47(4):271-277.

3. Alvord EC, Jr. Growth rates of epidermoid tumors. Ann Neurol. 1977;2(5):367-370.

4. Sabin HI, Bordi LT, Symon L. Epidermoid cysts and cholesterol granulomas centered on the posterior fossa: twenty years of diagnosis and management. Neurosurgery. 1987;21(6):798-805.

5. Altschuler EM, Jungreis CA, Sekhar LN, Jannetta PJ, Sheptak PE. Operative treatment of intracranial epidermoid cysts and cholesterol granulomas: report of 21 cases. Neurosurgery. 1990;26(4):606-613; discussion 614.

6. Lopes M, Capelle L, Duffau H, Kujas M, Sichez JP, Van Effenterre R, Faillot T, et al. [Surgery of intracranial epidermoid cysts. Report of 44 patients and review of the literature]. Neurochirurgie. 2002;48(1):5-13.

7. Chowdhury FH, Haque MR, Sarker MH. Intracranial epidermoid tumor; microneurosurgical management: An experience of 23 cases. Asian J Neurosurg. 2013;8(1):2128.

8. Del Bo L, Ambrosetti U. Hearing aids for the treatment of tinnitus. Prog Brain Res. 2007;166:341-345.

9. Eggermont JJ. On the pathophysiology of tinnitus; a review and a peripheral model. Hear Res. 1990;48(12):111-123.

10. Zhang Q, Kaga K, Sano M, Takegoshi H. Arachnoid cysts confined to the internal auditory canal or facial nerve canal. J Laryngol Otol. 2011;125(10):1053-1058. 
11. Kleinjung T, Steffens T, Strutz J, Langguth B. Curing tinnitus with a Cochlear Implant in a patient with unilateral sudden deafness: a case report. Cases J. 2009;2:7462.

12. Spansdahl T, Solheim O. Quality of life in adult patients with primary intracranial arachnoid cysts. Acta Neurochir
(Wien). 2007;149(10):1025-1032.

13. van der Meche FG, Braakman R. Arachnoid cysts in the middle cranial fossa: cause and treatment of progressive and non-progressive symptoms. J Neurol Neurosurg Psychiatry. 1983;46(12):1102-1107. 